\title{
Predicting mobility performance of a small, lightweight track system using the computer-aided method NTVPM
}

\author{
J.Y. Wong ${ }^{a,{ }^{*}}$, C. Senatore ${ }^{\text {b, }}$, P. Jayakumar ${ }^{c}$, and K. lagnemma ${ }^{b}$ \\ a Vehicle Systems Development Corporation, Ottawa, Ontario, Canada \\ ${ }^{b}$ Massachusetts Institute of Technology, Cambridge, MA, USA \\ ${ }^{\circ}$ U.S. Army TARDEC, Warren, MI, USA
}

\begin{abstract}
This paper describes the results of a study of applying the physics-based, computer-aided method - the Nepean Tracked Vehicle Performance Model (NTVPM), originally developed for evaluating the mobility of large, heavy tracked vehicles, to predicting the performance of a small, lightweight track system on sandy soil. The cross-country (tractive) performance of the track system predicted by NTVPM is compared with experimental data obtained in a laboratory soil bin by the Robotic Mobility Group, Massachusetts Institute of Technology. It is shown that the correlation between the tractive performance predicted by NTVPM and that measured is reasonably close, as indicated by the values of the coefficient of correlation, coefficient of determination, root mean squared deviation, and coefficient of variation. The results of this study provide evidence for supporting the view that physics-based methods, such as NTVPM, that are developed on the understanding of the physical nature and detailed analysis of vehicle-terrain interaction, are applicable to large, heavy, as well as small, lightweight vehicles, provided that appropriate terrain data are used as input.
\end{abstract}

Keywords: coefficient of correlation; coefficient of variation; computer-aided methods; tractive performance; experimental study; robotic vehicles; track systems.

\footnotetext{
${ }^{*}$ Corresponding author.

${ }^{* *}$ Present address: Exponent Inc., Natick, MA, USA.

E-mail addresses: vsdccanada@yahoo.ca (J.Y. Wong), csenatore@exponent.com (C. Senatore), paramsothy.jayakumar.civ@mail.mil (P. Jayakumar), and kdi@mit.edu (K. lagnemma).
} 


\section{Report Documentation Page}

Form Approved

OMB No. 0704-0188

Public reporting burden for the collection of information is estimated to average 1 hour per response, including the time for reviewing instructions, searching existing data sources, gathering and maintaining the data needed, and completing and reviewing the collection of information. Send comments regarding this burden estimate or any other aspect of this collection of information,

including suggestions for reducing this burden, to Washington Headquarters Services, Directorate for Information Operations and Reports, 1215 Jefferson Davis Highway, Suite 1204, Arlington

VA 22202-4302. Respondents should be aware that notwithstanding any other provision of law, no person shall be subject to a penalty for failing to comply with a collection of information if it

does not display a currently valid OMB control number.

1. REPORT DATE

24 APR 2015

4. TITLE AND SUBTITLE

Predicting mobility performance of a small, lightweight track system using the computer-aided method NTVPM

6. AUTHOR(S)

7. PERFORMING ORGANIZATION NAME(S) AND ADDRESS(ES)

US Army RDECOM-TARDEC,6501 E. 11 Mile

Road,Warren,MI,48397-5000

9. SPONSORING/MONITORING AGENCY NAME(S) AND ADDRESS(ES)

\section{DATES COVERED}

00-00-2015 to 00-00-2015

5a. CONTRACT NUMBER

5b. GRANT NUMBER

5c. PROGRAM ELEMENT NUMBER

5d. PROJECT NUMBER

5e. TASK NUMBER

5f. WORK UNIT NUMBER

8. PERFORMING ORGANIZATION REPORT NUMBER

10. SPONSOR/MONITOR'S ACRONYM(S)

11. SPONSOR/MONITOR'S REPORT $\operatorname{NUMBER}(\mathrm{S})$

12. DISTRIBUTION/AVAILABILITY STATEMENT

Approved for public release; distribution unlimited

13. SUPPLEMENTARY NOTES

14. ABSTRACT

See Report

15. SUBJECT TERMS

16. SECURITY CLASSIFICATION OF: a. REPORT

unclassified b. ABSTRACT

unclassified c. THIS PAGE

unclassified
17. LIMITATION OF ABSTRACT

Report (SAR)
Same as
18. NUMBER OF PAGES

24 19a. NAME OF RESPONSIBLE PERSON

$+2$


UNCLASSIFIED: Distribution Statement A. Approved for public release. \#26397

\section{Introduction}

Small, lightweight robotic vehicles have found increasingly wide applications in industrial, security, and defence operations, as well as extraterrestrial exploration. Quite often these vehicles are less than $1 \mathrm{~m}$ in length and weigh less $500 \mathrm{~N}$. They are normally equipped with wheels or tracks, while some with other forms of running gear, such as legs (limb-like appendages), have also been developed. In recent years, several small, lightweight robotic systems have adopted the track as running gear for improved cross-country mobility.

Studies of the dynamics of small, lightweight tracked vehicles have been reported. Watanabe et al. presented a model for the dynamic track-sand interaction (Watanabe et al., 1993). The turning behaviour of a small, lightweight tracked vehicle (with mass of $50.5 \mathrm{~kg}$, track contact length of $0.41 \mathrm{~m}$, and track width of $0.08 \mathrm{~m}$ ) predicted using the model was compared with experimental data. Negrut et al. investigated a computational framework for the physics-based simulation of lightweight tracked vehicles operating on discrete terrain (Negrut et al., 2013). As a demonstration of this technology, simulation results of a lightweight tracked vehicle negotiating obstacles, the dimensions of which were comparable to those of the vehicle, were presented. In these studies, the crosscountry performance of the vehicle was not examined.

This paper describes the results of a study of applying the physics-based, computeraided method NTVPM, originally developed for evaluating the mobility of large, heavy tracked vehicles (Wong, 2008; Wong 2010), to predicting the performance of a small, lightweight single track system on sandy soil. The cross-country performance of the track system predicted by NTVPM is compared with experimental data obtained in a laboratory soil bin by the Robotic Mobility Group, Massachusetts Institute of Technology (MIT) (Senatore et al., 2013).

\section{Computer-Aided Method NTVPM}

NTVPM is a physics-based, computer-aided method, developed by Vehicle Systems Development Corporation, Ottawa, Ontario, Canada. It is formulated on the understanding of the physical nature of vehicle-terrain interaction and on the detailed 
UNCLASSIFIED: Distribution Statement A. Approved for public release. \#26397

analysis of the mechanics of track-terrain interaction. It is for predicting the crosscountry performance of single-unit or two-unit articulated vehicles with either rubber band tracks or segmented metal tracks with relatively short track pitch. It is originally developed for evaluating the cross-country performance of large, heavy tracked vehicles, such as agricultural, industrial, and military tracked vehicles.

NTVPM takes into account all major vehicle design parameters that affect crosscountry performance. These include

(A) road wheel-track system configuration;

(B) suspension characteristics;

(C) initial track tension (i.e. the track tension when the vehicle is stationary on level, firm ground, which indicates whether the track is loose or tight) and track longitudinal stiffness.

Independent road wheel suspensions, including the torsional spring or translational spring type, with linear or nonlinear characteristics, can be simulated using NTVPM.

NTVPM also takes into consideration all pertinent terrain characteristics: the pressure-sinkage relationship, shear strength, and shear stress-shear displacement relationship of the terrain; rubber-terrain shearing characteristics (for vehicles with rubber band tracks or segmented metal tracks with rubber pads); and terrain response to repetitive normal and shear loading.

The output of NTVPM includes the normal and shear stress distributions on the trackterrain interface, track sinkage, external motion resistance, thrust, drawbar pull, and tractive efficiency as functions of track slip. The mean maximum pressure (MMP) and mean maximum shear stress under the track also form part of the output.

The capability of NTVPM for predicting the cross-country performance of heavy tracked vehicles over different types of terrain has been substantiated with field test data (Wong, 2008; Wong, 2010). It has also been successfully employed in assisting industry in the development of high-mobility tracked vehicles in various countries (Wong, 1992; Wong, 1995). For further information on the computer-aided method NTVPM, please refer to References (Wong, 2008; Wong, 2010). 
UNCLASSIFIED: Distribution Statement A. Approved for public release. \#26397

\section{Experimental Setup}

The single track system used in this study is shown in Figure 1 (Senatore et al., 2013). An aluminum frame encloses the drivetrain of the track system, while the suspension system is mounted externally. The drivetrain includes a motor assembly (with encoder, motor, and reduction gear), a flange-to-flange Futek TFF500 torque sensor, a flexible coupling, a one-to-one bevel gear set, and two toothed pulleys. Four road wheels, suspended with torsional springs on pivot arms, are attached to the track frame. The tensioner is located in the front and the sprocket at the rear. The track is made of rubber (a 92 shore A durometer toothed rubber belt produced by BrecoFlex). The track (belt) width is $100 \mathrm{~mm}$ and its contact length is approximately $250 \mathrm{~mm}$. The grousers (lugs) on the track are straight, with height of $5.5 \mathrm{~mm}$, width of $100 \mathrm{~mm}$, and thickness of $3 \mathrm{~mm}$ (in the longitudinal direction). The pitch between adjacent grousers is $33 \mathrm{~mm}$.

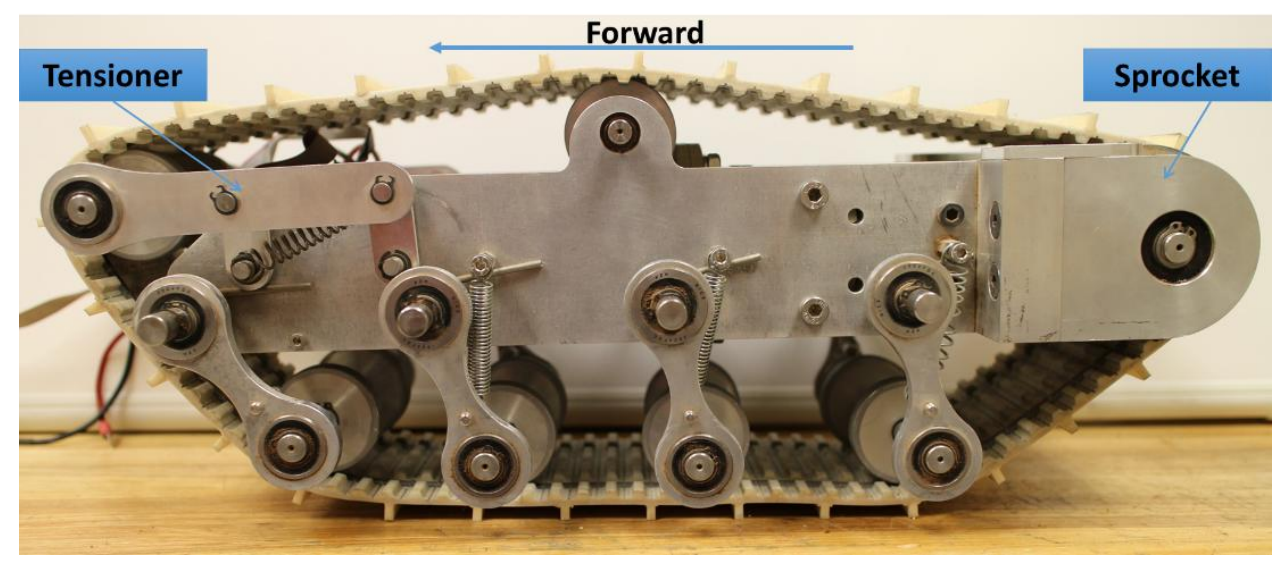

Figure 1. The single track system used in the study.

It should be mentioned that there is uncertainty in the values of the torsional stiffness of the springs of the road wheel suspensions and the free angular positions of the pivot arms (i.e. the angular positions of the pivot arms where the corresponding torsional springs are not subject to any load). These values required as input to NTVPM are therefore derived from a special procedure, which will be described in detail later.

The multipurpose test rig for measuring the performance of the track system is shown in Figure 2 (lagnemma et al., 2005). The track frame is attached to a mounting platform. An ATI Omega 85, 6-axis force/torque transducer is installed between the mounting 
platform and the carriage for measuring the vertical load on and the drawbar pull generated by the track system. The carriage slides on two low-friction rails to enable longitudinal translation of the track system. The track system can move freely in the vertical direction under various normal loads. The slip of the track is controlled by varying the angular speed of the sprocket in relation to the translational speed of the carriage. The horizontal movement of the carriage is controlled through a toothed belt driven by a $90 \mathrm{~W}$ Maxon DC motor, while the sprocket of the track is driven by a $150 \mathrm{~W}$ Maxon DC motor. The horizontal displacement of the carriage is measured by a Micro Epsilon WPS-1250-MK46 draw wire encoder. The maximum horizontal movement of the carriage is approximately $1 \mathrm{~m}$, with a maximum speed of approximately $120 \mathrm{~mm} / \mathrm{s}$ $(0.432 \mathrm{~km} / \mathrm{h})$.

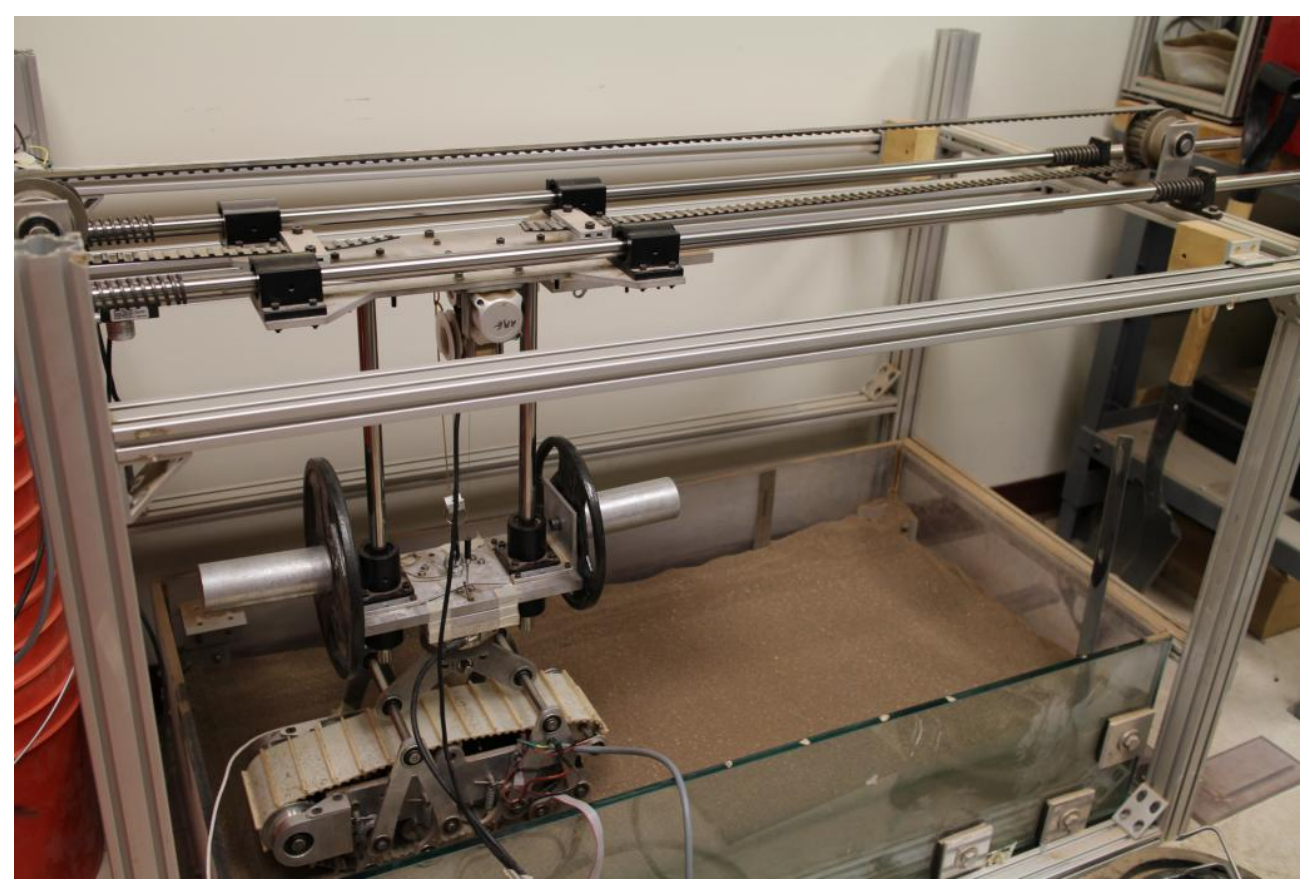

Figure 2. The multipurpose rig for testing the single track system.

It should be mentioned that the track frame was attached to the mounting platform with a fixed horizontal attitude and could not pitch freely (Senatore et al., 2013). It is unlike a track vehicle that can pitch freely under normal operating conditions. Consequently, the carriage must apply a constraining moment to maintain the track frame with a horizontal attitude during tests. This constraining moment, however, was 
UNCLASSIFIED: Distribution Statement A. Approved for public release. \#26397

not monitored during tests and is therefore unknown. To take this into account in the simulation of the performance of the track system using NTVPM, a special procedure was implemented. This ensured that in the simulation the track frame maintained a horizontal attitude, so that the performance predicted using NTVPM would be comparable to that measured experimentally. This special procedure used in the simulation will be described in detail later.

\section{Soil Properties}

Tests of the single track system were conducted in a soil bin of $1.5 \mathrm{~m}$ long, $0.7 \mathrm{~m}$ wide, and with soil depth of $0.16 \mathrm{~m}$. The soil used in the experiments was the Quikrete Medium Sand, which is silica sand with predominant grain size in the 0.3-0.8 $\mathrm{mm}$ range (Senatore et al., 2013).

The pressure-sinkage characteristics of the soil were measured using a bevameter with rectangular sinkage plates with length of $16 \mathrm{~cm}$ and widths of 3,5 , and $7 \mathrm{~cm}$. The maximum sinkage was approximately $3 \mathrm{~cm}$. The pressure-sinkage relationship of the soil was characterized using the Bekker equation (Bekker, 1969; Wong, 2008; Wong, 2010). The values of the pressure-sinkage parameters, $n, k_{c}$, and $k_{\phi}$, are given in Table 1 (Senatore et al., 2013).

The internal shearing characteristics of the soil were originally measured using a direct shear box, commonly used in civil engineering soil mechanics tests (Senatore et al., 2013). The shear stress-shear displacement relationship of the soil was characterized using the Janosi and Hanamoto equation (Janosi and Hanamoto, 1961). The values of the parameters obtained using the direct shear box were: cohesion $c=$ $1500 \mathrm{~Pa}$, angle of internal shearing resistance $\phi=34^{\circ}$, and shear deformation parameter $K=0.0006 \mathrm{~m}$ (Senatore, et al., 2013).

Since the track is made of rubber, shearing action takes place on the rubber grouser-soil interface, in addition to the internal soil shearing between adjacent rubber grousers. The parameters characterizing rubber-soil shearing are required as input to NTVPM for predicting the tractive performance of a rubber track system. However, the rubber-soil shearing tests were not conducted and the rubber-soil shearing data are not available. As a substitute, the rubber track of the single track system, with contact 
UNCLASSIFIED: Distribution Statement A. Approved for public release. \#26397

length of $250 \mathrm{~mm}$ and contact width of $100 \mathrm{~mm}$, was used as a linear shear device (hereinafter referred to as the track shear device) to obtain the appropriate values of the shear parameters. The values of the parameters so obtained represent the combined internal soil and rubber-soil shearing characteristics. Tests were performed under normal pressures of $1.5,2.3,4.8$, and $8.7 \mathrm{kPa}$. The average normal pressures on the single track system under normal loads of 125,155 , and $190 \mathrm{~N}$, at which the performance of the track system was measured, were $5,6.2$, and $7.6 \mathrm{kPa}$, respectively, thus falling into the range of normal pressures under which shear tests were performed. Using the track shear device, the values of the shear parameters derived from test data using the exact least-squares method (Senatore and lagnemma, 2011) were: cohesion $c=476 \mathrm{~Pa}$, angle of shearing resistance $\phi=28^{\circ}$, and shear deformation parameter $K=$ $0.0075 \mathrm{~m}$. This set of shear parameter values, which more realistically represents the rubber track-soil shearing behaviour than that obtained using the direct shear box, was used as input to NTVPM for predicting the performance of the single track system.

The parameters for characterizing the response of the terrain to repetitive normal loading are required as input to NTVPM. They are not, however, available for the Quikrete Medium Sand. For these parameters, estimated values based on those of similar sandy soil were used (Wong, 2008; Wong, 2010).

The values of the pressure-sinkage, shear, and repetitive loading parameters of the Quikrete Medium Sand, used as inputs to NTVPM for predicting the performance of the single track system, are summarized in Table 1.

Table 1

Parameters of the Quikrete Medium Sand used as input to NTVPM for predicting the performance of the single track system.

\begin{tabular}{|c|c|c|c|c|c|c|c|}
\hline \multicolumn{2}{|c|}{ Pressure-sinkage parameters } & \multicolumn{3}{c|}{ Shear parameters } & \multicolumn{2}{c|}{$\begin{array}{c}\text { Repetitive loading } \\
\text { parameters }\end{array}$} \\
\hline$n$ & $\begin{array}{c}k_{c} \\
\mathrm{kN} / \mathrm{m}^{\mathrm{n}+1}\end{array}$ & $\begin{array}{c}k_{\phi} \\
\mathrm{kN} / \mathrm{m}^{\mathrm{n} 2}\end{array}$ & $\begin{array}{c}c \\
\mathrm{~Pa}\end{array}$ & $\begin{array}{c}\phi \\
\mathrm{deg}\end{array}$ & $\begin{array}{c}K \\
\mathrm{~m}\end{array}$ & $\begin{array}{c}k_{o} \\
\mathrm{kN} / \mathrm{m}^{3}\end{array}$ & $\begin{array}{c}A_{u} \\
\mathrm{kN} / \mathrm{m}^{4}\end{array}$ \\
\hline 1 & -20 & 3130 & 476 & 28 & 0.0075 & 0 & 503,000 \\
\hline
\end{tabular}


UNCLASSIFIED: Distribution Statement A. Approved for public release. \#26397

\section{Simulation Setup}

5.1. Torsional stiffness of the springs and free angular positions of the pivot arms

As noted previously, there is uncertainty in the measurements of the torsional stiffness of the springs of the road wheel suspensions, and the free angular positions of the pivot arms of the single track system. To obtain reasonable values of the torsional stiffness of the springs and the free angular positions of the pivot arms for simulations using NTVPM, the procedure described below was followed.

(a) The single track system mounted on the carriage under test conditions was set up on level, firm ground;

(b) A normal load of $190 \mathrm{~N}$ was applied to the track system (as noted previously, in the tests, $190 \mathrm{~N}$ was one of the three normal loads applied to the single track system);

(c) The pivot arm angles of the four road wheels of the track system under normal load of $190 \mathrm{~N}$ on level, firm ground were measured, and the results are shown in Table 2;

(d) Simulations using NTVPM were conducted to replicate the pivot arm angles of the four road wheels of the track system measured under normal load of $190 \mathrm{~N}$ on level, firm ground, with various combinations of the torsional stiffness of the springs and the free angular positions of the pivot arms. The schematic of the single track system showing the pivot arm angular positions under load on level, firm ground, as part of the output of NTVPM, is presented in Figure 3. After a number of trial runs, it was found that the combination of the torsional stiffness of the springs and free angular positions of the pivot arms shown in Table 3 provides a reasonable match to the measured pivot arm angles shown in Table 2;

(e) With the combination of the torsional stiffness of the springs and free angular positions of the pivot arms shown in Table 3, the pivot arm angles of the four road wheels under normal load of $190 \mathrm{~N}$ on level, firm ground predicted by NTVPM are shown in Table 2. 
UNCLASSIFIED: Distribution Statement A. Approved for public release. \#26397

SINGLE TRACK SYSTEM

$\leftarrow$ Forward

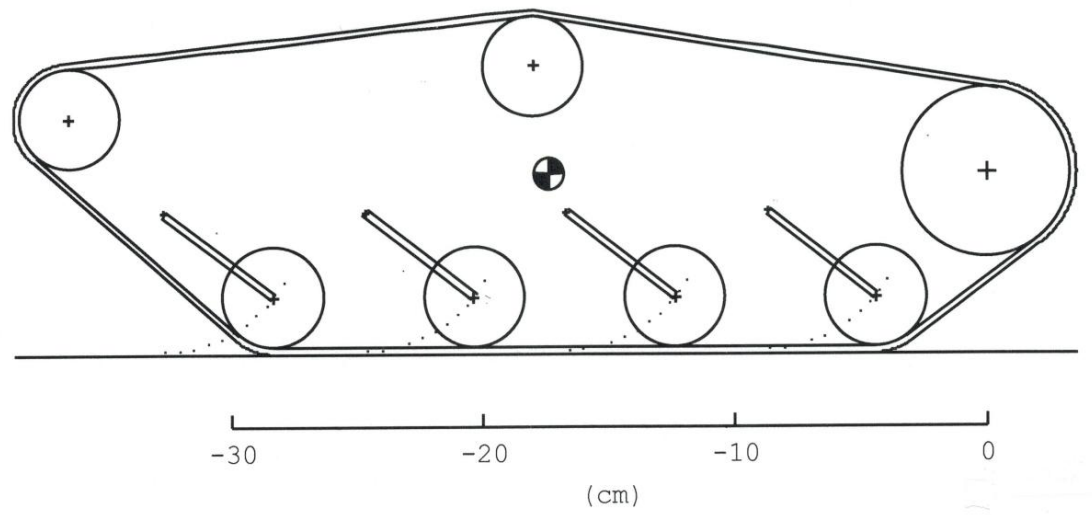

Figure 3. Schematic of the single track system on firm, level ground, showing the pivot arm angular positions under load, as part of the output of NTVPM.

Table 2

Comparison of the pivot arm angles predicted by NTVPM and the measured under normal load of $190 \mathrm{~N}$ on level, firm ground.

\begin{tabular}{|c|c|c|c|c|c|}
\hline \multirow{2}{*}{ Data type } & \multicolumn{5}{|c|}{ Pivot arm angles from the horizontal, deg. } \\
\hline & Wheel 1 & Wheel 2 & Wheel 3 & Wheel 4 & Average \\
\hline Measured & 37.35 & 36.38 & 38.85 & 39.64 & 38.06 \\
\hline $\begin{array}{l}\text { Predicted by } \\
\text { NTVPM }\end{array}$ & 37.60 & 37.93 & 38.25 & 38.58 & 38.09 \\
\hline \multirow[b]{2}{*}{ Deviation } & -0.25 & -1.55 & 0.60 & 1.06 & 0.03 \\
\hline & \multicolumn{5}{|c|}{$\begin{array}{l}\text { Root mean squared deviation } R M S D=0.99^{\circ} \text {; } \\
\text { Coefficient of variation } C V=0.026\end{array}$} \\
\hline
\end{tabular}

Table 3

Pivot arm free angular positions and torsional stiffness of the springs used as input to NTVPM for predicting the single track system performance.

\begin{tabular}{|c|c|c|c|c|}
\hline Parameters & Wheel 1 & Wheel 2 & Wheel 3 & Wheel 4 \\
\hline $\begin{array}{c}\text { Pivot arm free position angle from the } \\
\text { horizontal, deg. }\end{array}$ & 89 & 70 & 70 & 70 \\
\hline Torsional stiffness, kN-m/deg. & 0.00009 & 0.00008 & 0.00008 & 0.00008 \\
\hline
\end{tabular}

It can be seen from Table 2, the average of the four road wheel pivot arm angles measured under normal load of $190 \mathrm{~N}$ on level, firm ground is $38.06^{\circ}$, whereas with the 
UNCLASSIFIED: Distribution Statement A. Approved for public release. \#26397

combination of the torsional stiffness of the springs and free angular positions of the pivot arms shown in Table 3, the average of the four road wheel pivot arm angles predicted by NTVPM is $38.09^{\circ}$, a difference of $0.03^{\circ}$. The root mean squared deviation $R M S D$ between the predicted and the measured is $0.99^{\circ}$ and the coefficient of variation $C V$ (i.e. the ratio of the root mean squared deviation to the mean of the measured values of the four pivot arm angles) is 0.026 . These indicate that the combination of the torsional stiffness of the springs and the free angular positions of the pivot arms shown in Table 3 can be considered as a set of reasonable values for input to NTVPM for predicting the tractive performance of the single track system, in comparison with the measured performance obtained from tests.

\subsection{Maintaining the horizontal attitude of the track frame in simulations}

As noted earlier, the track frame was attached to the mounting platform in a fixed horizontal position. A constraining moment was therefore applied to the track frame during tests. This constraining moment was, however, not monitored during tests and is unknown. Using NTVPM to predict the performance of a track vehicle under normal operating conditions, the vehicle can pitch freely without constraint. To obtain simulated track performance at a given slip using NTVPM comparable to that obtained from tests, where the track frame was constrained to take a horizontal attitude, a special procedure described below was followed during simulations.

(a) Using NTVPM in predicting the performance of the track system at a given slip, the drawbar hitch vertical position was adjusted, until the track frame achieved a horizontal attitude (i.e. the angle between the track frame and the horizontal, referred to hereinafter as the trim angle, was equal or close to zero). This was equivalent to applying a constraining moment, created by the drawbar pull multiplied by the vertical distance of the drawbar hitch from the ground, to make the track frame exhibit a horizontal or close to horizontal attitude.

(b) At the particular drawbar hitch height, where the trim angle of the track frame was equal or close to zero, the performance at a given slip predicted by NTVPM was considered comparable to that obtained from tests at the corresponding slip. As an 
UNCLASSIFIED: Distribution Statement A. Approved for public release. \#26397

example, Figure 4 shows that the track frame achieves a zero trim angle at $30 \%$ slip under normal load of $190 \mathrm{~N}$ on the Quikrete Medium Sand.

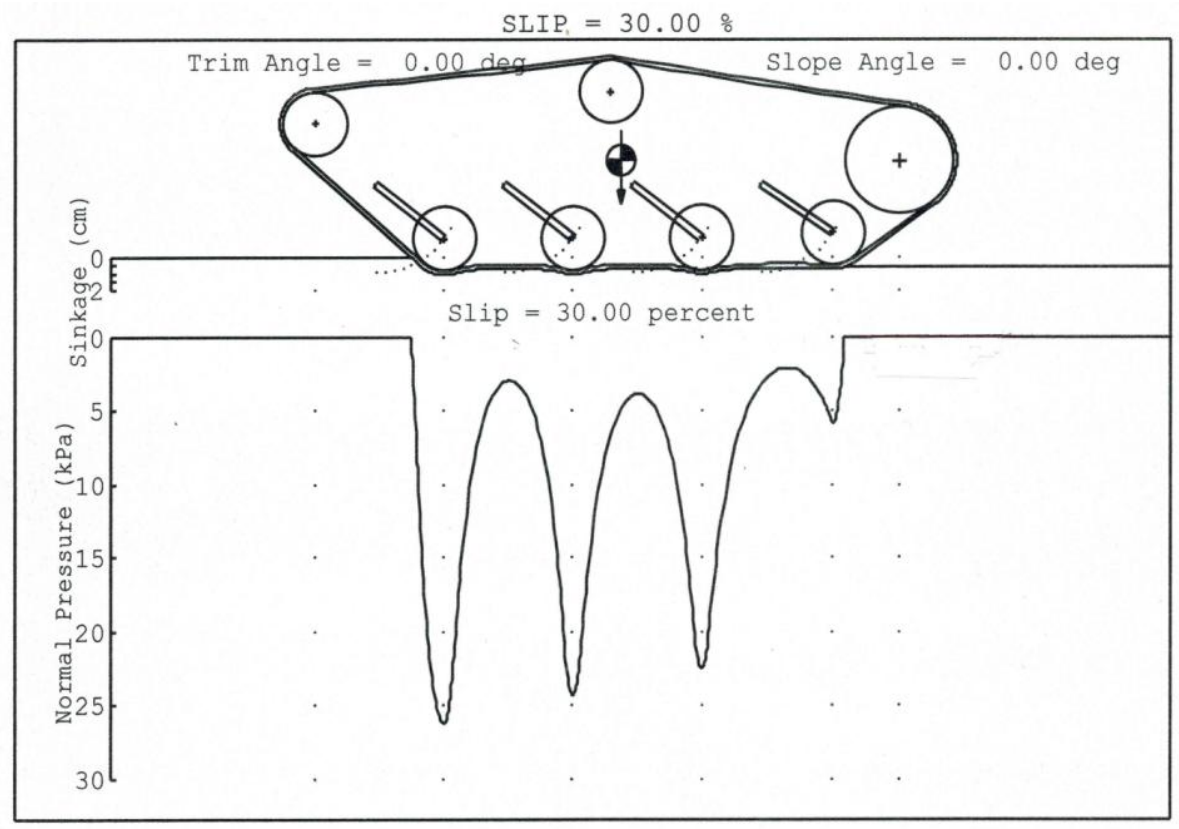

Figure 4. Zero trim angle of the track frame achieved at $30 \%$ slip under normal load of $190 \mathrm{~N}$, by adjusting the vertical position of the drawbar hitch.

\section{Correlations between the predicted performance and test data}

The performance of the single track system was predicted using NTVPM at slips of 10,30 , and $50 \%$ and under normal loads of 125,155 , and $190 \mathrm{~N}$ (including both the sprung and unsprung weight of the track system) on the Quikrete Medium Sand. The major design parameters of the single track system used as input to NTVPM are given in the Appendix. The terrain parameters used in the simulation are presented in Table 1.

\subsection{Under normal load $125 \mathrm{~N}$}

The values of the drawbar pull coefficient (i.e. the ratio of drawbar pull to normal load) of the track system under normal load of $125 \mathrm{~N}$ at slips of 10,30 , and $50 \%$ on Quikrete Medium Sand predicted by NTVPM, together with the corresponding mean values of the measured drawbar pull coefficient, are shown in Figure 5. The mean values of the measured drawbar pull coefficient plus one standard deviation and those minus one standard deviation at various slips, together with the predicted values, are 
UNCLASSIFIED: Distribution Statement A. Approved for public release. \#26397

shown in Figure 6. This illustrates the variability of the measured data in comparison to the predicted performance.

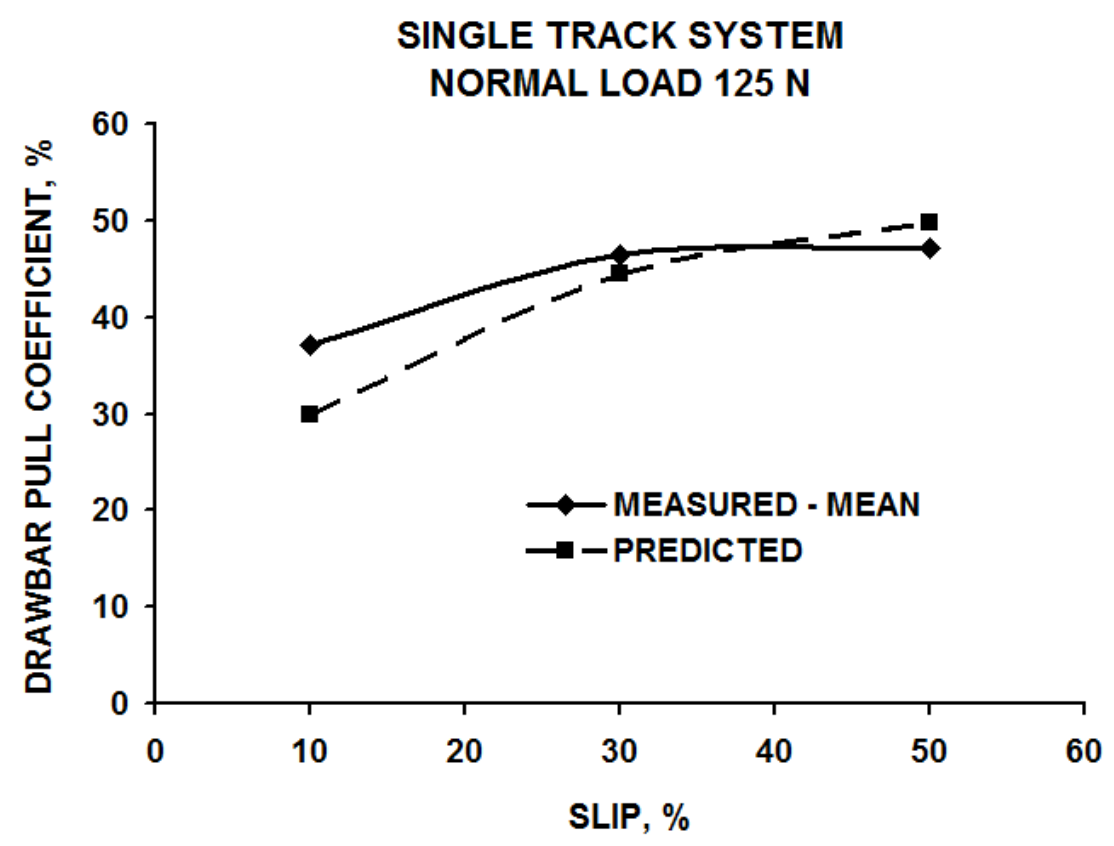

Figure 5. Comparison of the performance of the single track system under load of $125 \mathrm{~N}$ predicted by NTVPM with the mean measured performance.

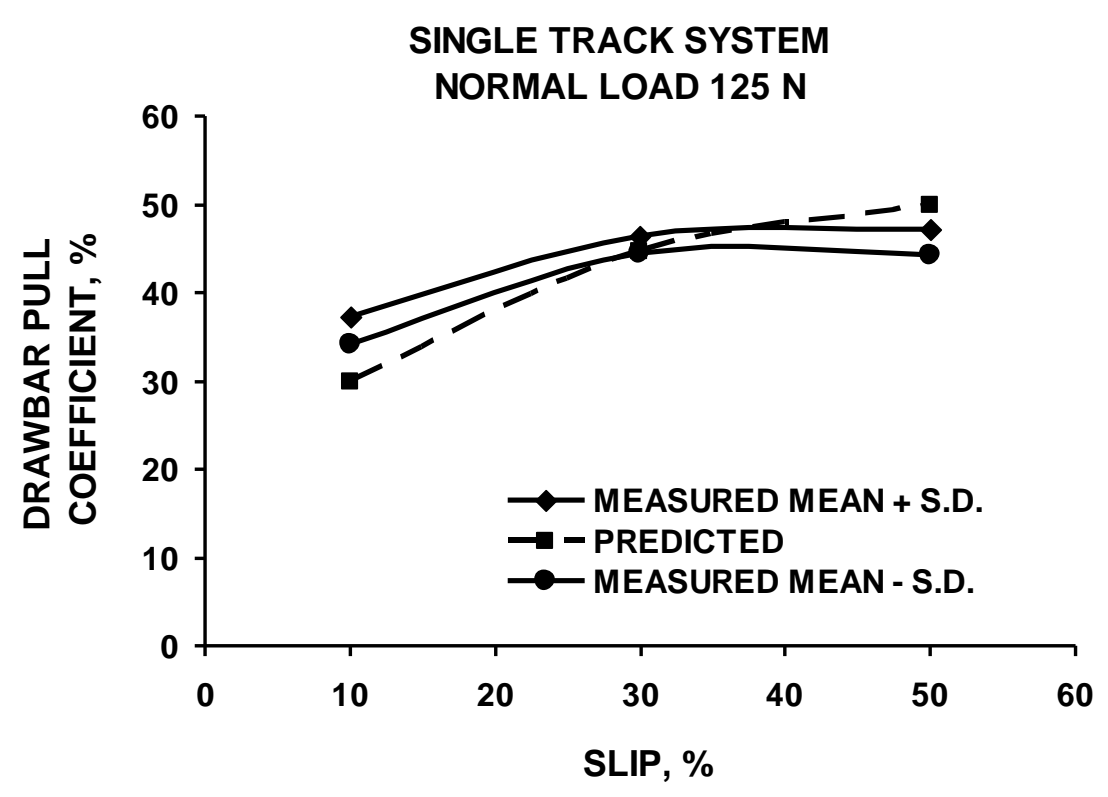

Figure 6. Comparison of the performance of the single track system under load of $125 \mathrm{~N}$ predicted by NTVPM with the mean measured performance with +/- one standard devaition. 
UNCLASSIFIED: Distribution Statement A. Approved for public release. \#26397

The predicted and measured drawbar pull coefficient-slip relationships shown together in Figures 5 and 6 are useful for visual comparison of the degree of their correlation.

To quantitatively evaluate the overall correlation between the performance predicted by NTVPM and the measured data, two criteria are adopted in this study. Firstly, the coefficient of correlation $R$ and the associated coefficient of determination $R^{2}$ are used to evaluate the correlation between the trends of the drawbar pull coefficient-slip relationship predicted by NTVPM and those measured. Secondly, the root mean squared deviation $R M S D$ and the coefficient of variation $C V$ (i.e. the ratio of $R M S D$ to the mean of measured values) are used to quantitatively evaluate the deviations between the values of the drawbar pull coefficient predicted by NTVPM and the mean values of the measured at corresponding slips.

(A) The coefficient of correlation $R$ and the coefficient of determination $R^{2}$

The coefficient of correlation $R$ is defined as

$$
R=\frac{n \sum x y-\left(\sum x\right)\left(\sum y\right)}{\left[\sqrt{n\left(\sum x^{2}\right)-\left(\sum x\right)^{2}}\right]\left[\sqrt{n\left(\sum y^{2}\right)-\left(\sum y\right)^{2}}\right]}
$$

where $x$ and $y$ represent the predicted and measured drawbar pull coefficient at the corresponding slips, respectively; and $n$ is the number of data points used in the evaluation.

A value of one (1) for the coefficient of correlation $R$ indicates a perfect correlation between the trends of the predicted and measured data. The correlation will generally be regarded as strong if the value of $R$ is greater than 0.8 . With a value of $R$ less than 0.5 , the correlation is usually regarded as weak. The coefficient of determination $R^{2}$ (the square of the coefficient of correlation $R$ ) gives the proportion of the variance of one variable that is predictable from the other. For example, if $R^{2}=0.85$, it indicates that $85 \%$ of the variation in one variable will be predictable from the other. In other words, $R^{2}$ is a measure that determines how certain one can be in making predictions from a particular model.

(B) The root mean squared deviation $R M S D$ and the coefficient of variation $C V$ 
UNCLASSIFIED: Distribution Statement A. Approved for public release. \#26397

The root mean squared deviation $R M S D$ is defined as

$R M S D=\sqrt{\frac{\sum(x-y)^{2}}{n}}$

where $x, y$ and $n$ are the same as those in Equation (1).

If the value of $R M S D$ is zero, the predicted and measured data will have a perfect match, with zero deviations between them. It should be noted that the RMSD has the same unit as $x$ and $y$. For this particular case, it is in the same unit as that of drawbar pull coefficient. To provide a general indicator for the degree of match between the predicted and measured data, the coefficient of variation $C V$, a non-dimensional parameter, is introduced, as mentioned previously.

$C V=R M S D / Y$

where $Y$ is the mean value of the measured drawbar pull coefficient.

Table 4 shows the values of the predicted and measured drawbar pull coefficient at various slips of the single track system under normal load of $125 \mathrm{~N}$, and the values of the coefficient of correlation $R$, coefficient of determination $R^{2}$, root mean squared deviation $R M S D$, and coefficient of variation $C V$. It should be noted that in the calculations, the mean values of the measured drawbar pull coefficient are used.

As can be seen from Table 4 , the values of $R$ and $R^{2}$ are 0.972 and 0.945 , respectively. Thus, the correlation between the trends of the drawbar pull coefficient-slip relationship predicted by NTVPM and those of the measured can be regarded as strong. The average of the ratios of the mean of the measured drawbar pull coefficient to the predicted by NTVPM at various slips is 1.043 . The values of RMSD and CV are $4.13 \%$ and 0.098 , respectively. Thus, the deviations between the predicted and the measured can be regarded as reasonable.

It should be noted from column 3 of Table 4, the predicted values of the drawbar pull coefficient at various slips are obtained with the trim angle of the track frame being zero. Thus, as explained in Section 5, the performance predicted by NTVPM and the corresponding measured are comparable, as they are obtained under the same operating conditions, that is, the track frame both during tests and in simulations maintains a horizontal attitude. 
UNCLASSIFIED: Distribution Statement A. Approved for public release. \#26397

Table 4

Correlation between the drawbar pull coefficient of the single track system under normal load of $125 \mathrm{~N}$ predicted by NTVPM and the measured on Quikrete Medium Sand.

\begin{tabular}{|c|c|c|c|c|c|c|c|c|}
\hline \multirow{2}{*}{$\begin{array}{c}\text { Normal } \\
\text { load } \\
\mathrm{N}\end{array}$} & \multirow{2}{*}{$\begin{array}{l}\text { Slip } \\
\%\end{array}$} & \multirow{2}{*}{$\begin{array}{c}\text { Trim } \\
\text { angle* } \\
\text { deg. }\end{array}$} & \multicolumn{4}{|c|}{ Drawbar pull coefficient, \% } & \multirow[b]{2}{*}{$\begin{array}{c}\text { Measured } \\
\text { mean/ } \\
\text { predicted } \\
\text { by } \\
\text { NTVPM }\end{array}$} & \multirow[b]{2}{*}{$\begin{array}{c}\text { Deviation } \\
\text { (measured } \\
\text { mean- } \\
\text { predicted) } \\
\%\end{array}$} \\
\hline & & & $\begin{array}{l}\text { Measured } \\
\text { mean }\end{array}$ & $\begin{array}{l}\text { Measured } \\
\text { Mean }+ \text { SD }\end{array}$ & $\begin{array}{l}\text { Measured } \\
\text { Mean - SD }\end{array}$ & $\begin{array}{c}\text { Predicted } \\
\text { by } \\
\text { NTVPM }\end{array}$ & & \\
\hline \multirow{5}{*}{125} & 10 & 0.00 & 35.68 & 37.2 & 34.16 & 29.91 & 1.193 & 5.77 \\
\hline & 30 & 0.00 & 45.44 & 46.4 & 44.48 & 44.57 & 1.020 & 0.87 \\
\hline & 50 & 0.00 & 45.68 & 47.12 & 44.24 & 49.83 & 0.917 & -4.15 \\
\hline & \multicolumn{6}{|c|}{ Average } & 1.043 & $\begin{array}{c}R M S D= \\
4.13\end{array}$ \\
\hline & \multicolumn{8}{|c|}{$\begin{array}{c}\text { Coefficient of correlation } R=0.972 ; \text { Coefficient of determination } R^{2}=0.945 ; \\
\text { Coefficient of variation } C V=0.098\end{array}$} \\
\hline
\end{tabular}

${ }^{*}$ Trim angle of the track frame in simulations by NTVPM.

\subsection{Under normal load $155 \mathrm{~N}$}

The values of the drawbar pull coefficient of the track system under normal load of $155 \mathrm{~N}$ at slips of 10,30 , and $50 \%$ on Quikrete Medium Sand predicted by NTVPM, together with the corresponding mean values of the measured drawbar pull coefficient are shown in Figure 7. The mean values of the measured drawbar pull coefficient plus one standard deviation and those minus one standard deviation at various slips, together with the predicted values, are shown in Figure 8 . This illustrates the variability of the measured data in comparison to the predicted performance. 
UNCLASSIFIED: Distribution Statement A. Approved for public release. \#26397

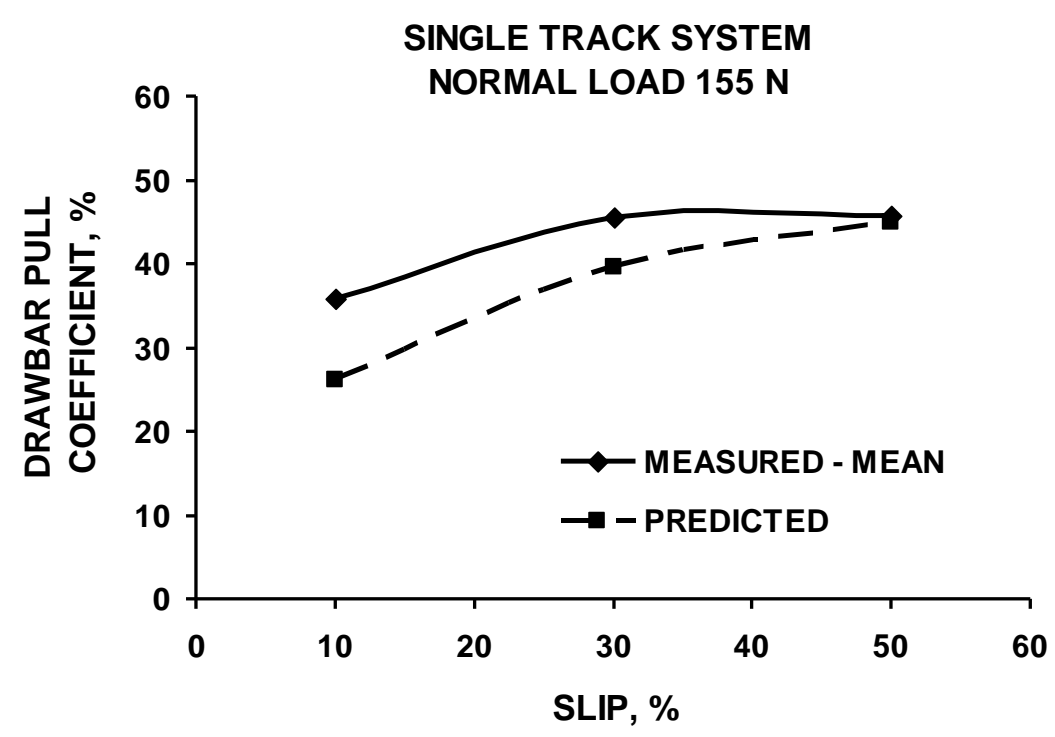

Figure 7. Comparison of the performance of the single track system under load of $155 \mathrm{~N}$ predicted by NTVPM with the mean measured performance.

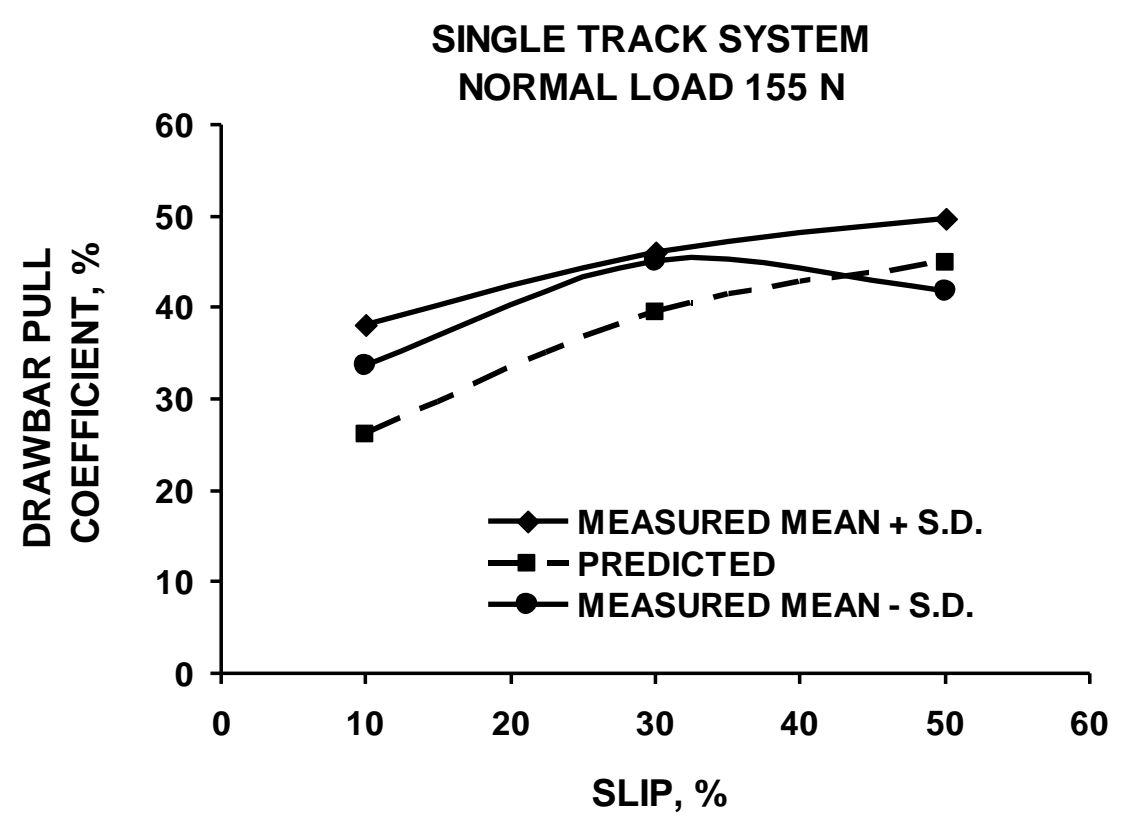

Figure 8. Comparison of the performance of the single track system under load of $155 \mathrm{~N}$ predicted by NTVPM with the mean measured performance with $+/$ - one standard devaition.

Table 5 shows the values of the predicted and measured drawbar pull coefficient at various slips of the single track system under normal load of $155 \mathrm{~N}$, as well as the values of the coefficient of correlation $R$, coefficient of determination $R^{2}$, root mean 
UNCLASSIFIED: Distribution Statement A. Approved for public release. \#26397

squared deviation $R M S D$, and coefficient of variation $C V$. It should be noted that in the calculations, the mean values of the measured drawbar pull coefficient are used.

Table 5

Correlation between the drawbar pull coefficient of the single track system under normal load of 155 N predicted by NTVPM and the measured on Quikrete Medium Sand.

\begin{tabular}{|c|c|c|c|c|c|c|c|c|}
\hline \multirow{2}{*}{$\begin{array}{l}\text { Normal } \\
\text { load } \\
\mathrm{N}\end{array}$} & \multirow{2}{*}{$\begin{array}{l}\text { Slip } \\
\%\end{array}$} & \multirow{2}{*}{$\begin{array}{c}\text { Trim } \\
\text { angle* } \\
\text { deg. }\end{array}$} & \multicolumn{4}{|c|}{ Drawbar pull coefficient, \% } & \multirow[b]{2}{*}{$\begin{array}{c}\text { Measured } \\
\text { mean/ } \\
\text { predicted } \\
\text { by } \\
\text { NTVPM }\end{array}$} & \multirow[b]{2}{*}{$\begin{array}{c}\text { Deviation } \\
\text { (measured } \\
\text { mean- } \\
\text { predicted) } \\
\%\end{array}$} \\
\hline & & & $\begin{array}{l}\text { Measured } \\
\text { mean }\end{array}$ & $\begin{array}{c}\text { Measured } \\
\text { Mean + SD }\end{array}$ & $\begin{array}{l}\text { Measured } \\
\text { Mean - SD }\end{array}$ & $\begin{array}{c}\text { Predicted } \\
\text { by } \\
\text { NTVPM }\end{array}$ & & \\
\hline \multirow{5}{*}{155} & 10 & 0.00 & 35.87 & 38.13 & 33.61 & 26.21 & 1.369 & 9.66 \\
\hline & 30 & 0.00 & 45.61 & 46.06 & 45.16 & 39.51 & 1.154 & 6.1 \\
\hline & 50 & 0.00 & 45.81 & 49.81 & 41.81 & 44.87 & 1.021 & 0.94 \\
\hline & \multicolumn{6}{|c|}{ Average } & 1.181 & $\begin{array}{c}\text { RMSD }= \\
6.62\end{array}$ \\
\hline & \multicolumn{8}{|c|}{$\begin{array}{c}\text { Coefficient of correlation } R=0.965 ; \text { Coefficient of determination } R^{2}=0.931 ; \\
\text { Coefficient of variation } C V=0.156 \text {. }\end{array}$} \\
\hline
\end{tabular}

${ }^{*}$ Trim angle of the track frame in simulations by NTVPM.

As can be seen from Table 5, the values of $R$ and $R^{2}$ are 0.965 and 0.931 , respectively. Thus, the correlation between the trends of the predicted drawbar pull coefficient-slip relationship by NTVPM and those of the measured can be regarded as strong. The average of the ratios of the mean of the measured drawbar pull coefficient to the predicted by NTVPM at various slips is 1.181 . The values of $R M S D$ and $C V$ are $6.62 \%$ and 0.156 , respectively. Thus, the deviations between the predicted and the measured can be regarded as reasonable.

It should be noted from column 3 of Table 5 that the predicted values of the drawbar pull coefficient at various slips are obtained with the trim angle of the track frame being zero. Thus, as explained in Section 5, the predicted performance by NTVPM and the corresponding measured are comparable, as they are obtained under the same operating conditions, that is, the track frame both during tests and in simulations maintains a horizontal attitude. 
UNCLASSIFIED: Distribution Statement A. Approved for public release. \#26397

\subsection{Under normal load $190 \mathrm{~N}$}

The values of the drawbar pull coefficient of the track system under normal load of $190 \mathrm{~N}$ at slips of 10,30 , and $50 \%$ on Quikrete Medium Sand predicted by NTVPM, together with the corresponding mean values of the measured drawbar pull coefficient are shown in Figure 9. . The mean values of the measured drawbar pull coefficient plus one standard deviation and those minus one standard deviation at various slips, together with the predicted values, are shown in Figure 10. This illustrates the variability of the measured data in comparison to the predicted performance.

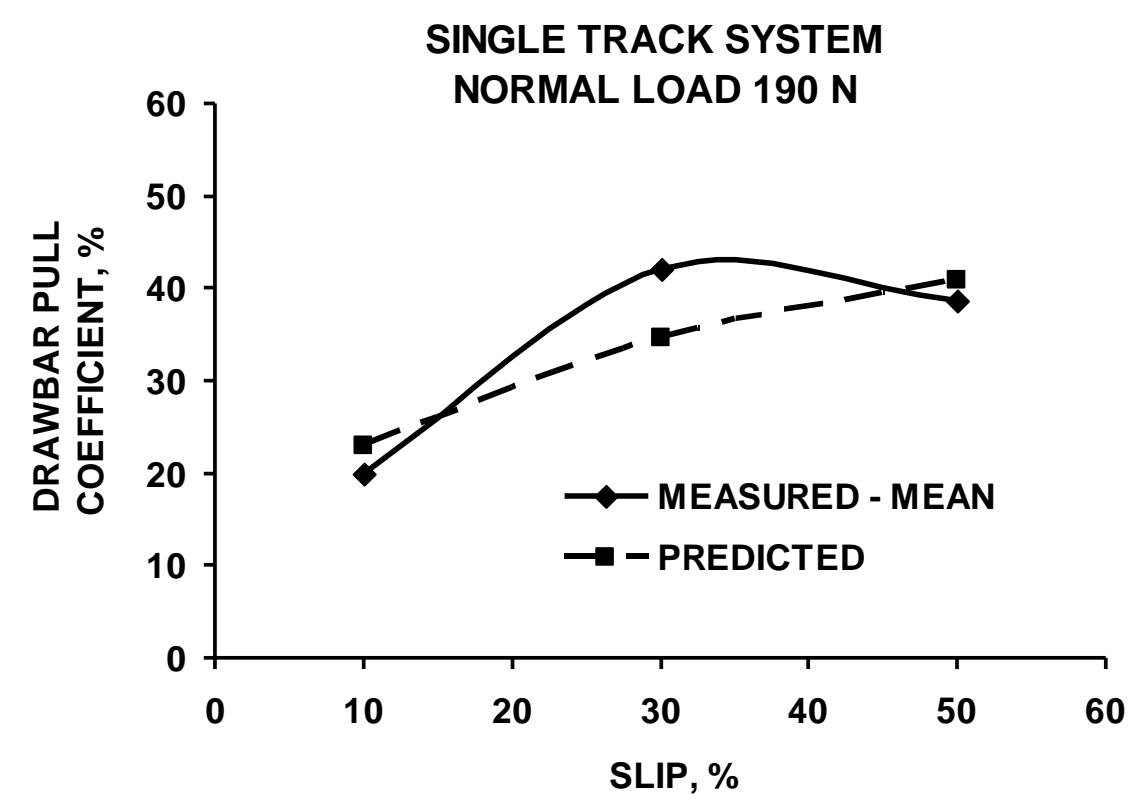

Figure 9. Comparison of the performance of the single track system under load of $190 \mathrm{~N}$ predicted by NTVPM with the mean measured performance. 


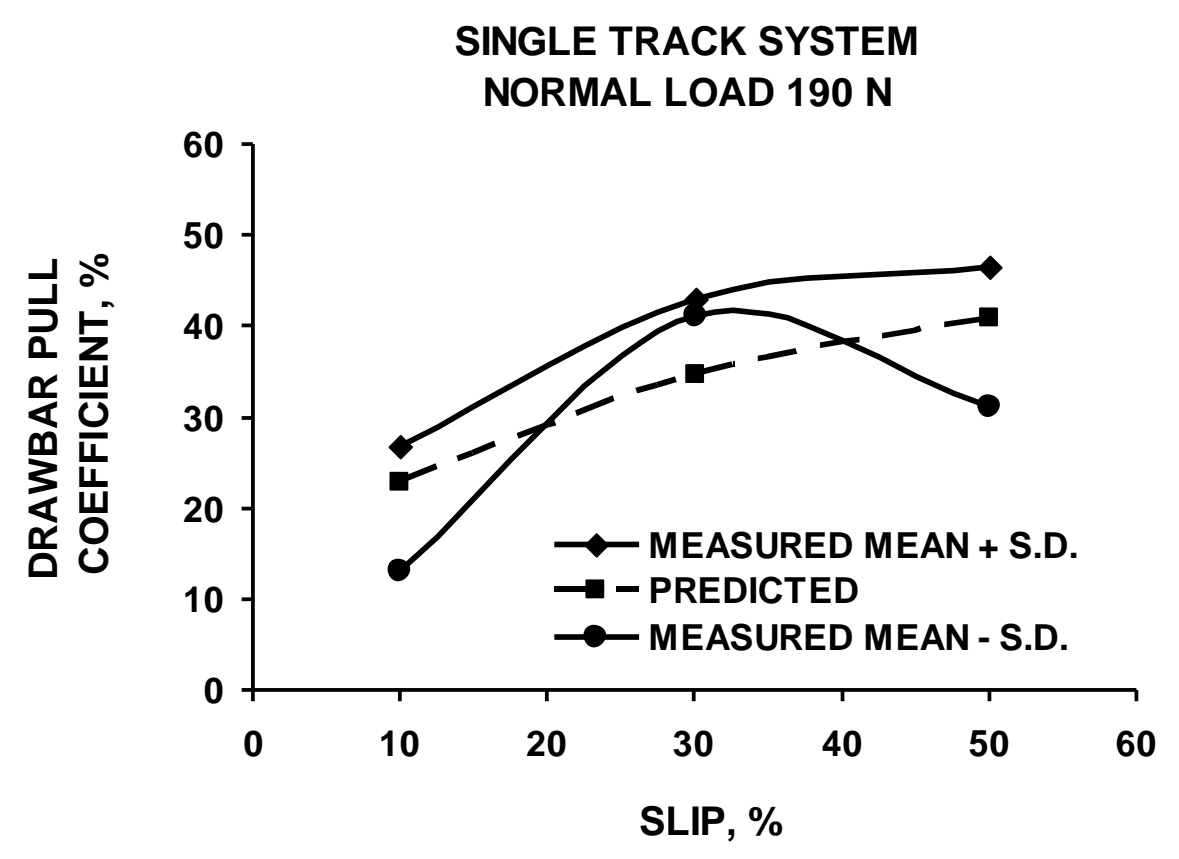

Figure 10. Comparison of the performance of the single track system under load of $190 \mathrm{~N}$ predicted by NTVPM with the mean measured performance with +/- one standard devaiation.

Table 6 shows the values of the predicted and measured drawbar pull coefficient at various slips of the single track system under normal load of $190 \mathrm{~N}$, and the values of the coefficient of correlation $R$, coefficient of determination $R^{2}$, root mean squared deviation $R M S D$, and coefficient of variation $C V$. It should be noted that in the calculations, the mean values of the measured drawbar pull coefficient are used.

As can be seen from Table 6 , the values of $R$ and $R^{2}$ are 0.904 and 0.817 , respectively. Thus, the correlation between the trends of the predicted drawbar pull coefficient-slip relationship by NTVPM and those of the measured can be regarded as strong. The average of the ratios of the mean of the measured drawbar pull coefficient to the predicted by NTVPM at various slips is 1.010. The values of RMSD and $C V$ are $4.63 \%$ and 0.137 , respectively. Thus, the deviations between the predicted and the measured can be regarded as reasonable. 
UNCLASSIFIED: Distribution Statement A. Approved for public release. \#26397

Table 6

Correlation between the drawbar pull coefficient of the single track system under normal load of $190 \mathrm{~N}$ predicted by NTVPM and the measured on Quikrete Medium Sand.

\begin{tabular}{|c|c|c|c|c|c|c|c|c|}
\hline \multirow{2}{*}{$\begin{array}{l}\text { Normal } \\
\text { load } \\
\mathrm{N}\end{array}$} & \multirow{2}{*}{$\begin{array}{c}\text { Slip } \\
\%\end{array}$} & \multirow{2}{*}{$\begin{array}{c}\text { Trim } \\
\text { angle* } \\
\text { deg. }\end{array}$} & \multicolumn{4}{|c|}{ Drawbar pull coefficient, \% } & \multirow[b]{2}{*}{$\begin{array}{c}\text { Measured } \\
\text { mean/ } \\
\text { predicted } \\
\text { by } \\
\text { NTVPM }\end{array}$} & \multirow[b]{2}{*}{$\begin{array}{c}\text { Deviation } \\
\text { (measured } \\
\text { mean- } \\
\text { predicted) } \\
\%\end{array}$} \\
\hline & & & $\begin{array}{c}\text { Measured } \\
\text { mean }\end{array}$ & $\begin{array}{c}\text { Measured } \\
\text { Mean + SD }\end{array}$ & $\begin{array}{l}\text { Measured } \\
\text { Mean - SD }\end{array}$ & $\begin{array}{c}\text { Predicted } \\
\text { by } \\
\text { NTVPM }\end{array}$ & & \\
\hline \multirow{5}{*}{190} & 10 & +0.02 & 19.95 & 26.79 & 13.11 & 22.90 & 0.871 & -2.95 \\
\hline & 30 & 0.00 & 42.05 & 43.05 & 41.05 & 34.68 & 1.213 & 7.37 \\
\hline & 50 & +0.01 & 38.74 & 46.42 & 31.05 & 40.90 & 0.947 & -2.16 \\
\hline & \multicolumn{6}{|c|}{ Average } & 1.010 & $\begin{array}{c}R M S D= \\
4.63\end{array}$ \\
\hline & \multicolumn{8}{|c|}{$\begin{array}{c}\text { Coefficient of correlation } R=0.904 ; \text { Coefficient of determination } R^{2}=0.817 \text {; } \\
\text { Coefficient of variation } C V=0.137\end{array}$} \\
\hline
\end{tabular}

${ }^{*}$ Trim angle of the track frame in simulations by NTVPM. Positive trim angle indicates the track frame taking a nose-up attitude.

It should be noted from column 3 of Table 6 that the predicted values of the drawbar pull coefficient at various slips are obtained with trim angles of the track frame being $+0.02^{\circ}, 0.00^{\circ}$, and $+0.01^{\circ}$ at slips of 10,30 , and $50 \%$, respectively (positive trim angle indicates the track frame taking a nose-up attitude). While at slips of 10 and $50 \%$, the trim angles are not zero, they are very small. Thus, as explained in Section 5, the predicted performance by NTVPM and the corresponding measured are comparable, as they are obtained under similar operating conditions.

\section{Closing remarks}

(A) A study of the correlation between the tractive performance of a small, lightweight single track system on sandy soil predicted by NTVPM and that measured in experiments has been conducted. NTVPM is a physics-based, computer-aided method and is originally developed for predicting the cross-country performance of large, heavy tracked vehicles, such as agricultural, industrial, and military tracked vehicles. It is shown that the correlation between the trends of the drawbar pull coefficient-slip relationships predicted by NTVPM and those measured is reasonably close, as indicated by the values of the coefficient of correlation $R$ and coefficient of determination $R^{2}$. For instance, under normal load of $125 \mathrm{~N}$, the value of $R$ is 0.972 and that of $R^{2}$ is 
UNCLASSIFIED: Distribution Statement A. Approved for public release. \#26397

0.945. As is generally accepted, a value of $R$ greater than 0.8 signifies a strong correlation. The value of the root mean squared deviation $R M S D$ is $4.13 \%$ and that of the coefficient of variation $C V$ is 0.098 . Similar results are obtained with the track system under normal loads of 155 and $190 \mathrm{~N}$. These indicate that the correlation between the tractive performance predicted by NTVPM and that measured can be regarded as reasonable.

(B) There is a preconception that methods developed for predicting the performance of large, heavy off-road vehicles may not be applicable to predicting the performance of small, lightweight vehicles. For empirically-based methods, this could be the case. As is well known, empirical relations, in general, may not be extrapolated beyond the conditions upon which they are derived. For physics-based methods, such as NTVPM, which are developed on the understanding of the physical nature and detailed analysis of vehicle-terrain interaction, they are applicable to large and heavy, as well as small and lightweight vehicles, provided that appropriate terrain data are used as input, and the fundamental mechanisms of soil deformation and failure remain similar across scales. The results of this study provide evidence for supporting this view, as it is demonstrated that there is a reasonably close correlation between the performance of the small, lightweight track system predicted by NTVPM and measured data. A study of applying the physics-based, computer-aided method - the Nepean Wheeled Vehicle Performance Model (NWVPM), originally developed for predicting the mobility of large, heavy off-road wheeled vehicles, to predicting the performance of lunar roving vehicle wheels led to similar findings (Wong and Asnani, 2008).

\section{Acknowledgements}

The prediction of the track system performance using NTVPM and the analysis of the correlations between the predicted and measured performance presented in this paper were performed under the auspices of Vehicle Systems Development Corporation (VSDC), Ottawa, Ontario, Canada. The experimental data of track performance used in this paper were from the Reference (Senatore, Jayakumar, and lagnemma, 2013). The study from which the experimental data were produced was conducted by the Robotic Mobility Group of the Massachusetts Institute of Technology 
UNCLASSIFIED: Distribution Statement A. Approved for public release. \#26397

(MIT) and supported by the U.S. Army Tank Automotive Research, Development and Engineering Center (TARDEC) and the Army Research Office (ARO) under award number W911NF-13-1-0063.

\section{Disclaimer}

Reference herein to any specific commercial company, product, process or service by trade name, trademark, manufacturer, or otherwise, does not necessarily constitute or imply its endorsement, recommendation, or favouring by the United States Government or the Department of the Army (DoA). The opinions of the authors expressed herein do not necessarily state or reflect those of the United States Government or the DoA, and shall not be used for advertising or product endorsement purposes. 
UNCLASSIFIED: Distribution Statement A. Approved for public release. \#26397

\section{References}

1. Bekker, M.G., 1969. Introduction to terrain-vehicle systems. University of Michigan Press, Ann Arbor, Michigan.

2. lagnemma, K., Shibly, H., and Dubowsky, S., 2005. A laboratory single wheel testbed for studying planetary rover wheel-terrain interaction. MIT Field and Space Robotic Laboratory Technical Report, 1:05-05.

3. Janozi, Z. and Hanamoto, B., 1961. The analytical determination of drawbar pull as a function of slip for tracked vehicles in deformable soils. In: Proceedings of the $1^{\text {st }}$ International Conference on the Mechanics of Soil-Vehicle Systems, Torino, Italy.

4. Negrut, D., Melanz, D., Mazhar, H., Lamb, D., Jayakumar, P., Letherwood, M., 2013. Investigating through simulation the mobility of light tracked vehicles operating on discrete granular terrain. SAE International J Passenger Cars -Mech. Syst. May, 6:369-381.

5. Senatore, C., and lagnemma, K., 2011. Direct shear behaviour of dry, granular soils for low normal stress with application to lightweight robotic vehicle modelling. In: Proceedings of the $17^{\text {th }}$ ISTVS International Conference, Blacksburg, VA, USA.

6. Senatore, C., Jayakumar, P., lagnemma, K., 2013. Experimental study of lightweight tracked vehicle performance on dry granular materials. In: Proceedings of ISTVS $7^{\text {th }}$ Americas Regional Conference, Tampa, FL, USA.

7. Watanabe, K., Murakami, H., Kitano, M., and Katahira, T., 1993. Experimental characterization of dynamic soil-track interaction on dry sand. J Terramechanics, 30, 111-131.

8. Wong, J.Y., 1992. Optimization of the tractive performance of articulated tracked vehicles using an advanced computer simulation model. J Automobile Engineering, Proceedings of the Institution of Mechanical Engineers, Part D. 206, 29-45.

9. Wong, J.Y., 1995. Application of the computer simulation model NTVPM-86 to the development of a new version of the infantry fighting vehicle ASCOD. J Terramechanics. 32, 53-61.

10. Wong, J.Y., 2008. Theory of ground vehicles, $4^{\text {th }}$ edition. John Wiley, New York.

11. Wong, J.Y., 2010. Terramechanics and off-road vehicle engineering, $2^{\text {nd }}$ edition. Elsevier, Oxford, England.

12. Wong, J.Y. and Asnani V.M., 2008. Study of the correlation between the performances of lunar vehicle wheels predicted by the Nepean wheeled vehicle performance model and test data. J Automobile Engineering, Proceedings of the Institution of Mechanical Engineers, Part D. 222, 1939-1954. 
UNCLASSIFIED: Distribution Statement A. Approved for public release. \#26397

\section{Appendix}

Major design parameters of the single track system

Sprung weight

$0.116,0.146$, or $0.181 \mathrm{kN}$

Unsprung weight

$0.009 \mathrm{kN}$

Sprung weight center of gravity $\mathrm{x}$-coordinate*

$-17.38 \mathrm{~cm}$

Sprung weight center of gravity y-coordinate*

$0 \mathrm{~cm}$

Initial track tension

$0.078 \mathrm{kN}$

Fixed wheel data

\begin{tabular}{|c|c|c|c|}
\hline Wheel type & $\begin{array}{l}\text { Wheel radius } \\
\mathrm{cm}\end{array}$ & $\begin{array}{c}\text { Wheel center } \\
\text { x-coordinate }{ }^{*}, \mathrm{~cm}\end{array}$ & $\begin{array}{l}\text { Wheel center } \\
y \text {-coordinate* }, \mathrm{cm}\end{array}$ \\
\hline Sprocket & 3.35 & 0.0 & 0.0 \\
\hline Tensioner & 2.0 & -36.40 & -2.30 \\
\hline
\end{tabular}

Road wheel suspensions

\begin{tabular}{|c|c|c|c|c|c|}
\hline $\begin{array}{c}\text { Wheel } \\
\text { radius } \\
\mathrm{cm}\end{array}$ & $\begin{array}{c}\text { Pivot arm } \\
\text { center } \\
\text { x-coord. } \\
\text { cm }\end{array}$ & $\begin{array}{c}\text { Pivot arm } \\
\text { center } \\
\text { y-coord. } \\
\text { cm }\end{array}$ & $\begin{array}{c}\text { Torsional } \\
\text { stiffness, } \\
\text { kN-m/deg. }\end{array}$ & $\begin{array}{c}\text { Pivot arm } \\
\text { free } \\
\text { position } \\
\text { angle, deg. }\end{array}$ & $\begin{array}{c}\text { Pivot arm } \\
\text { length, } \\
\mathrm{cm}\end{array}$ \\
\hline 2.0 & -32.7 & 1.5 & 0.00009 & 89 & 5.5 \\
\hline 2.0 & -24.7 & 1.5 & 0.00008 & 70 & 5.5 \\
\hline 2.0 & -16.7 & 1.5 & 0.00008 & 70 & 5.5 \\
\hline 2.0 & -8.7 & 1.5 & 0.00008 & 70 & 5.5 \\
\hline
\end{tabular}

Supporting roller

\begin{tabular}{|c|c|c|}
\hline Roller radius, $\mathrm{cm}$ & Roller center $\mathrm{x}$-coord. ${ }^{*}, \mathrm{~cm}$ & Roller center $\mathrm{y}$-coord. ${ }^{*}, \mathrm{~cm}$ \\
\hline 2.0 & -18.0 & -4.3 \\
\hline
\end{tabular}

Track parameters

Weight per unit length

$0.0053 \mathrm{kN} / \mathrm{m}$

Width

$10 \mathrm{~cm}$

Pitch

$3.3 \mathrm{~cm}$

Height of grouser

$0.55 \mathrm{~cm}$

Longitudinal stiffness (tension/elongation in \%)

$2200 \mathrm{kN}$

${ }^{\star}$ Coordinate origin is at the center of sprocket. Positive $\mathrm{x}$ - and $\mathrm{y}$-coordinates are to the rear and down, respectively. 